Article

\title{
Analysis and Mitigation of Crosstalk Effect on Coastal GNSS-R Code-Level Altimetry Using L5 Signals from QZSS GEO
}

\author{
Yunqiao He ${ }^{1}\left(\mathbb{D}\right.$, Tianhe $\mathrm{Xu}^{2, *}$, , Fan Gao ${ }^{1,2}$, Nazi Wang ${ }^{2}$, Xinyue Meng ${ }^{1}$ and Baojiao Ning ${ }^{1}$ \\ 1 School of Space Science and Physics, Shandong University, Weihai 264209, China; \\ heyunqiao@mail.sdu.edu.cn (Y.H.); gaofan@sdu.edu.cn (F.G.); 201916534@mail.sdu.edu.cn (X.M.); \\ 202017724@mail.sdu.edu.cn (B.N.) \\ 2 Institute of Space Sciences, Shandong University, Weihai 264209, China; wnz@sdu.edu.cn \\ * Correspondence: thxu@sdu.edu.cn
}

Citation: He, Y.; Xu, T.; Gao, F.; Wang, N.; Meng, X.; Ning, B. Analysis and Mitigation of Crosstalk Effect on Coastal GNSS-R Code-Level Altimetry Using L5 Signals from QZSS GEO. Remote Sens. 2021, 13, 4553. https://doi.org/10.3390/ rs13224553

Academic Editor: Kegen Yu

Received: 3 October 2021

Accepted: 10 November 2021

Published: 12 November 2021

Publisher's Note: MDPI stays neutral with regard to jurisdictional claims in published maps and institutional affiliations.

Copyright: (c) 2021 by the authors. Licensee MDPI, Basel, Switzerland. This article is an open access article distributed under the terms and conditions of the Creative Commons Attribution (CC BY) license (https:/ / creativecommons.org/licenses/by/ $4.0 /)$.

\begin{abstract}
Coastal Global Navigation Satellite System Reflectometry (GNSS-R) can be used as a valuable supplement for conventional tide gauges, which can be applied for marine environment monitoring and disaster warning. Incidentally, an important problem in dual-antenna GNSS-R altimetry is the crosstalk effect, which means that the direct signal leaks into the down-looking antenna dedicated to the reflected signals. When the path delay between the direct and reflected signals is less than one chip length, the delay waveform of the reflected signal is distorted, and the code-level altimetry precision decreases consequently. To solve this problem, the author deduced the influence of signal crosstalk on the reflected signal structure as the same as the multipath effect. Then, a simulation and a coastal experiment are performed to analyze the crosstalk effect on code delay measurements. The L5 signal transmitted by the Quasi-Zenith Satellite System (QZSS) from a geosynchronous equatorial orbit (GEO) satellite is used to avoid the signal power variations with the elevation, so that high-precision GNSS-R code altimetry measurements are achieved in the experiment. Theoretically and experimentally, we found there exists a bias in proportion to the power of the crosstalk signals and a high-frequency term related to the phase delay between the direct and reflected signals. After weakening the crosstalk by correcting the delay waveform, the results show that the RMSE between 23-h sea level height (SSH) measurements and the in-situ observations is about $9.5 \mathrm{~cm}$.
\end{abstract}

Keywords: altimetry; crosstalk effect; GNSS-R; GEO; L5

\section{Introduction}

The tide gauges (TG) located along continental coastlines and islands are crucial for providing global sea surface height $(\mathrm{SSH})$ variation. However, the SSH data are directly affected by land vertical motions, and there is a decreasing number of TGs [1-3]. The Global Navigation Satellite System Reflectometry (GNSS-R) was proposed to retrieve SSH by using the signals of opportunity transmitted by the GNSS satellites [4]. It has the potential to become a low-cost coastal monitoring system that can provide high-precision, high spatial-temporal resolution measurements and work under all-weather conditions. In general, three different approaches are used to process GNSS reflected signals: the GNSS interferometric reflectometry (GNSS-IR), the interferometric GNSS-R (iGNSS-R), and the conventional GNSS-R (cGNSS-R). GNSS-IR takes advantage of the interference phenomenon, known as the multipath effect when the direct signal and the reflected signal enter the same geodetic antenna at low elevation angles [5-8]. A 10-year individual water level estimation showed a root mean square error (RMSE) of about $12 \mathrm{~cm}$ [9]. However, the temporal resolution is low because the sampling rate is fundamentally limited by the number of satellite overflights and elevation angle spans [10]. Compared with GNSS-IR, iGNSS-R and cGNSS-R adopt two antennas and have been proven to be feasible methods for remote sensing in a variety of experiments, such as ground-based, shipborne, airborne, 
and space-borne [11-15]. In iGNSS-R, the direct and reflected signals are directly correlated, which allows using the entire signal bandwidth to improve the range solution [16]. The enhancement in altimetry precision of iGNSS-R is 2-6 times that of cGNSS-R in 1-s integrated measurements [17]. However, more complex hardware than cGNSS-R is required to conquer the drawbacks of reduced signal-to-noise ratio (SNR) and larger vulnerability to radio frequency interference (RFI) signals [18]. In cGNSS-R, the reflected GNSS signal is crosscorrelated with a clean replica of the transmitted code, which is beneficial for the coastal experiment [19]. The analysis in this paper is, therefore, based on the cGNSS-R method.

In dual antenna GNSS-R architectures, the direct and reflected signals are received by dedicated antennas. However, these signals easily enter the same antenna due to the inappropriate polarization isolation of the antennas. When the line-of-sight (Los) direct signal enters the antenna dedicated to the reflected signal, the reflected signal becomes distorted, which is called signal crosstalk [20]. Particularly, when the receiver height is low, the delay between the reflected signal and the direct signal is less than one code chip and it is hard to distinguish between them in the delay waveform (DW). This results in a declination of the code delay accuracy. An error of standard deviation up to $40 \mathrm{~cm}$ is found caused by the crosstalk effect for an airborne iGNSS-R experiment. [21]. Statistical analysis of the crosstalk in iGNSS-R shows that for elevation angles higher than $60^{\circ}$, crosstalk can be almost permanent from the ground up to $61 \%$ from airborne receivers at $2-\mathrm{km}$ height [18]. Some new estimation approaches are proposed to mitigate the crosstalk in ground-based and low altitude airborne GNSS-R [20]. Moreover, the impact of signal crosstalk on coastal GNSS-R altimetry using signals from GEO satellites has not been analyzed. Similar to multipath in standard GNSS receivers, the code, carrier phase, and SNR are also affected by the crosstalk. For the coastal code-level altimetry, biases for both B1C and B2a are found for each satellite by using the in-situ SSH [22]. Hence, $10 \mathrm{~cm}$ of bias is observed between the real data and the mean estimate by means of code and phase delay integration [23]. For the carrier phase altimetry, high accuracy is achieved using the signals from the QZSS GEO [24]. Obviously, the crosstalk impact on GNSS-R code delay altimetry is more severe. Incidentally, code-level measurements are important observations in coastal GNSS-R altimetry and the crosstalk impact has to be considered.

With the development of the GNSS, more new-generation civil signals have appeared. L1 and L5 are the shared frequency points of BDS-3 (B1C, B2a), GPS (L1, L5), Galileo (E1, E5), and other systems [25]. As multi-GNSS satellites are available, higher spatial and temporal resolution can be achieved [26]. Nevertheless, the code rate of L1 C/A is $1.023 \mathrm{MHz}$, which leads to poor altimetry performance due to the long code chip. L5 has greater bandwidth for improved jam resistance, four times stronger transmission power, and 10 times faster code rate than those of L1, and therefore a better GNSS-R code altimetry performance in the coastal experiment is expected $[27,28]$. In general, the crosstalk signals change with the elevation angle variation of GNSS satellites, showing the same signal structure as the direct signals. Thus, it is difficult to analyze the impact of the crosstalk effect on the observations because of the satellite's motion. The non-coherent averaging method is used to weak the crosstalk. In order to simplify the problem, Quasi-Zenith Satellite System (QZSS) is adopted. QZSS is an augmentation system to enhance the Global Positioning System (GPS) in the Asia-Oceania regions. It transmits signals that are compatible with the GPS L1C/A signal, as well as the modernized GPS L1C, L2C, and L5 signals [29]. At present, the services of one Geostationary Earth Orbit (GEO) satellite and three Quasi-Zenith Satellite Orbit (QZO) satellites are available. With a stable elevation angle of the GEO satellite, it would be convenient to extract the effect of the crosstalk from the observations. Hence, L5 signals from the QZSS GEO satellite are selected to analyze the influence of the crosstalk on coastal GNSS-R code measurements.

In this paper, the direct signals were collected as a reference to correlate with the reflected signals in order to generate the power waveform based on the modification of SDRLIB [30]. We firstly derived and simulated the signal crosstalk impact on the code delay measurements with QZSS GEO satellites. Then, a coastal experiment using L5 signals 
from the QZSS GEO satellite is carried out to verify the analysis. The crosstalk is weakened according to the simulation and experiment, and altimetry performance is analyzed.

The organization of this paper is as follows. In Section 2, the principle of coastal code altimetry is explained, and the influence of signal crosstalk on the code delay measurements is derived and simulated. In Section 3, an introduction to the experiment and an analysis of the effect of signal crosstalk on the code delay measurements are provided. Moreover, the altimetry results after the signal crosstalk elimination are shown. Finally, Sections 4 and 5 provide a discussion and conclusion of the paper, respectively.

\section{Methods}

\subsection{Code Delay Altimetry}

In wind speed retrieval, Zavorotny and Voronovich established the power model between GNSS reflected signals and sea surface characteristics based on the bistatic radar equation and geometric optics method of Kirchhoff approximation [31]. The model indicated that the reflected GNSS power was affected by the sea conditions, the propagation path, and the geometry. Meanwhile, in the GNSS-R receiver, the reflected GNSS signal power waveform is the cross-correlation of the reflected signals and local signals at different time delay $\tau$ under specific Doppler frequency shift $f_{0}$ as below [32]:

$$
\begin{gathered}
Y\left(\tau, t_{0}\right)=\int_{0}^{T_{i}} s_{R}\left(t_{0}+t^{\prime}+\tau\right) c\left(t_{0}+t^{\prime}\right) \exp \left[2 \pi j\left(f_{I F}+f_{0}\right)\left(t_{0}+t^{\prime}\right)\right] d t^{\prime} \\
P\left(\tau, t_{0}\right)=\int_{0}^{T_{a}}\left|Y\left(\tau, t_{0}\right)\right|^{2} d t
\end{gathered}
$$

where $Y\left(\tau, t_{0}\right)$ is the correlation value. $P\left(\tau, t_{0}\right)$ is the correlation power. $s_{R}$ is the reflected signal. $c$ is the local code replica at epoch $t_{0} . f_{I F}$ is the center frequency. $T_{i}$ is the coherent integration time. $T_{a}$ is the non-coherent integration time. To improve the signal-to-noise ratio, a large number of incoherent averages are required to obtain the correlation power. Figure 1 shows the correlation power of the direct and the reflected signals under the coastal conditions.

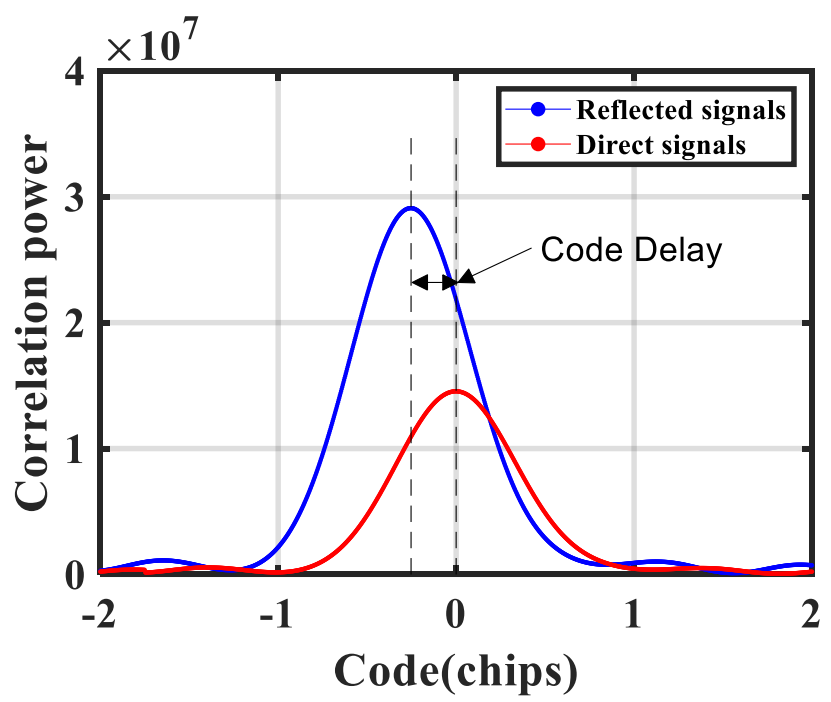

Figure 1. The correlation power waveform of the reflected (blue) and direct signals (red) in coastal conditions.

From the distance between the peak positions of the two waveforms, the code path delay is obtained and can be expressed as:

$$
\Delta \rho=\left(\rho^{\mathrm{r}}-\rho^{\mathrm{d}}\right)+\left(\rho_{\mathrm{a}}^{\mathrm{r}}-\rho_{\mathrm{a}}^{d}\right)+\rho_{\text {rough }}^{\mathrm{r}}+\rho_{\text {ins }}^{\mathrm{r}}+\epsilon^{\mathrm{rd}}
$$


where $\Delta \rho$ is the code path delay observations, $\rho^{\mathrm{r}}$ and $\rho^{\mathrm{d}}$ are the code measurements of the direct and reflected signals, respectively. $\rho_{\mathrm{a}}^{\mathrm{r}}$ and $\rho_{\mathrm{a}}^{d}$ are the atmosphere-induced delays, including tropospheric delay and ionospheric delay. $\rho_{\text {rough }}^{r}$ is the surface-roughnessinduced term. $\rho_{\text {ins }}^{\mathrm{r}}$ is the possible instrumental bias. $\epsilon^{\text {rd }}$ is the unmodelled noise. Under the coastal condition, most of the atmospheric contributions to the delay will be common to both branches, and thus they cancel one another out. The errors due to the roughness of the sea surface, the instrument, and unknown reasons can be weakened by filtering [33].

Figure 2 illustrates the GNSS-R altimetry geometry in the coastal condition, and the sea surface height (SSH) can be calculated as:

$$
\mathrm{SSH}=\mathrm{H}_{r}-\frac{\Delta \rho-\vec{d} \cdot \vec{e}}{2 \sin \theta}
$$

where $\mathrm{H}_{r}$ is the geodetic height of the reflector, which can be acquired by the positioning of the up-looking GNSS antenna. $\theta$ is the elevation angle of the GNSS satellites, $\vec{d}$ is the vector between the up-looking and down-looking antennae, and $\vec{e}$ is the unit vector between the reflector and the GNSS satellite. GNSS-R altimetry can provide a high-precision SSH by measurements of the vertical land motion. As shown in (3), the accuracy of the SSH depends on the path delay measurements between the direct and the reflected signals.

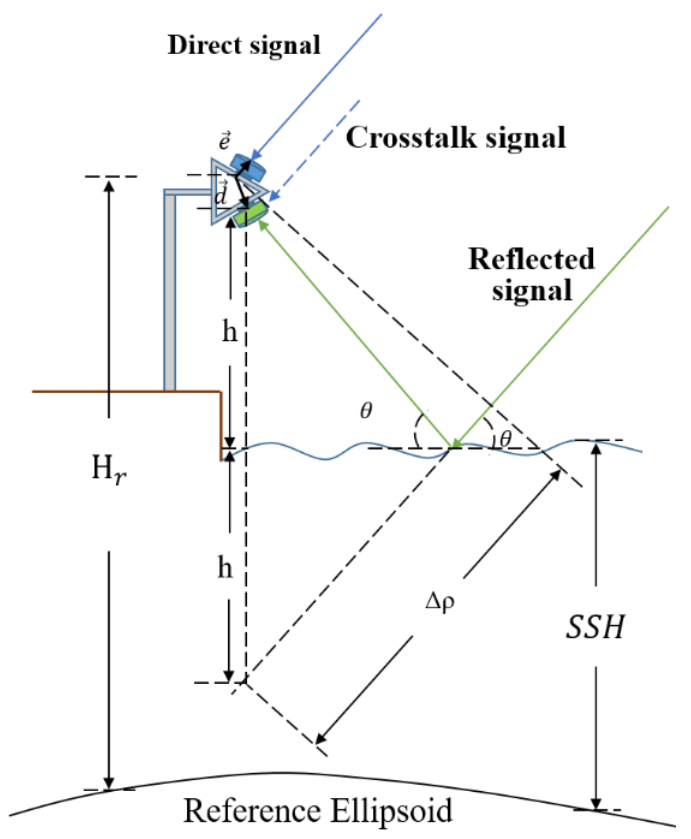

Figure 2. The geometry of coastal GNSS-R altimetry.

\subsection{Signal Crosstalk}

Figure 2 also shows the signal crosstalk in GNSS-R altimetry in the coastal condition. When only considering the specular reflection path, the crosstalk signal, the direct signal, and the reflected signal can be given as bellow $[34,35]$ :

$$
\left\{\begin{array}{c}
S_{c}=A_{c} \sin \left[2 \pi f t+C(t) \times \pi+\phi_{m}\right] \\
S_{d}=A_{d} \sin \left[2 \pi f t+C(t) \times \pi+\phi_{m}\right] \\
S_{r}=A_{r} \sin \left[2 \pi f(t+\delta t)+C(t+\delta t) \times \pi+\phi_{m}\right]
\end{array}\right.
$$

where $A_{c}, A_{d}$, and $A_{r}$ are the amplitudes of the crosstalk, direct, and reflected, respectively, $f$ is the frequency of the carrier phase, $\delta t$ is the time delay between the direct and the reflected signals, and $C(t)$ is the binary sequences including the ranging code and the 
navigation message. The binary sequences are transmitted by phase modulation, which causes phase reversal and limits the coherent integration time. $\phi_{m}$ is the initial phase of the direct signal. To facilitate analysis, the binary sequences of the reflected signals can be rewritten as:

$$
C(t+\delta t)=C(t)+\Delta c(t)
$$

The signals received by the down-looking antennae are the synthetic signals of the crosstalk signals and reflected signals, shown as:

$$
\mathrm{S}_{\mathrm{syn}}=S_{\mathrm{c}}+S_{r}
$$

Then, according to (5) and (6), Equation (7) can be expressed as:

$$
\begin{gathered}
\mathrm{S}_{\text {syn }}=A \sin \left(2 \pi f t+C(t) \times \pi+\phi_{m}+\beta\right), \\
A=\sqrt{A_{c}^{2}+A_{r}^{2}+2 \cdot A_{c} \cdot A_{r} \cos (\delta \phi),} \\
\beta=\arctan \frac{\frac{A_{c}}{A_{d}} \sin (\delta \phi+\Delta c(t) \times \pi)}{1+\frac{A_{c}}{A_{d}} \cos (\delta \phi+\Delta c(t) \times \pi)},
\end{gathered}
$$

where $A$ and $\beta$ are the amplitude and phase of the synthetic signal and $\delta \phi$ is the phase path delay between the direct and reflected signals, where $\Delta c(t)$ that can be eliminated for the determined value in (6). Similar to the analysis of the multipath effect, the impact of the crosstalk effect on the code delay measurements is greater than that of the phase delay [36], especially when $\frac{A_{c}}{A_{d}} \ll 1$. In the processing of the reflected signal, the direct signals are used to generate a continuously updated reference signal, which is given as:

$$
S_{r e f}=\sin \left[2 \pi f t+C(t) \times \pi+\phi_{m}\right]
$$

The complex waveform consists of the in-phase $I(t)$ and quadrature signal components $Q(t)$ are obtained after the correlation of the reflected signal and the reference signal.

$$
\left\{\begin{array}{l}
I(t, \tau)=\frac{A}{2} \cdot D(t) \operatorname{sinc}(\delta t+\tau) R(\delta t+\tau) \cos (\delta \phi)+\eta_{I} \\
Q(t, \tau)=\frac{A}{2} \cdot D(t) \sin c(\delta t+\tau) R(\delta t+\tau) \sin (\delta \phi)+\eta_{Q}
\end{array},\right.
$$

where $D(t)$ is the navigation bits and $R(\delta t+\tau)$ is the correlation value. The power waveform after $\mathrm{N}-\mathrm{ms}$ non-coherent integration can be expressed as:

$$
\operatorname{Power}(\tau, t)=\sum_{1}^{N}\left(I^{2}+Q^{2}\right)
$$

The power waveform is linked to three parts, which are $A_{c}^{2}, A_{r}^{2}$, and $2 \cdot A_{m} \cdot A_{r} \cos (\delta \phi)$. These variables are used to refer to the related parts in the power waveform hereafter. According to (3), only the part related to $A_{r}^{2}$ is the power waveform of the reflected signals. The power waveform of the synthetic signal has changed in relation to the reflected signal due to the crosstalk. Figure 3 shows the power waveform of the reflected signals, the crosstalk signals, and the synthetic signals at epoch $t$. The precision of the code delay observations is decreased as a result of the delay error caused by the crosstalk. In addition, the bias cannot be mitigated by the non-coherent averaging method given the approximately constant value. 


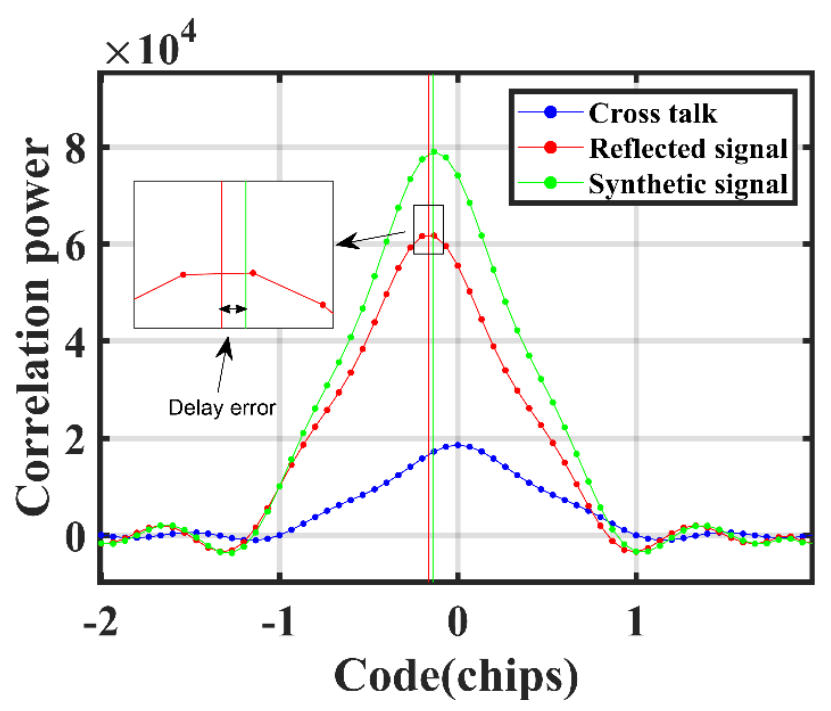

Figure 3. The delay map of the crosstalk signal, the reflected signal, and the synthetic signal.

\section{Results}

\subsection{Experiment}

In order to verify the above analysis, a coastal GNSS-R code altimetry experiment was carried out on a trestle in Weihai city, Shandong Province, China $\left(37^{\circ} 32^{\prime} 2.60^{\prime \prime} \mathrm{N}\right.$, $122^{\circ} 2^{\prime} 44.06^{\prime \prime} \mathrm{E}$ ) from 5 to 6 November 2020. The top view of the experiment is shown in Figure 4. The GNSS-R reflector is installed at the end of the trestle, about $100 \mathrm{~m}$ away from the coastline, effectively avoiding the influence of the land.

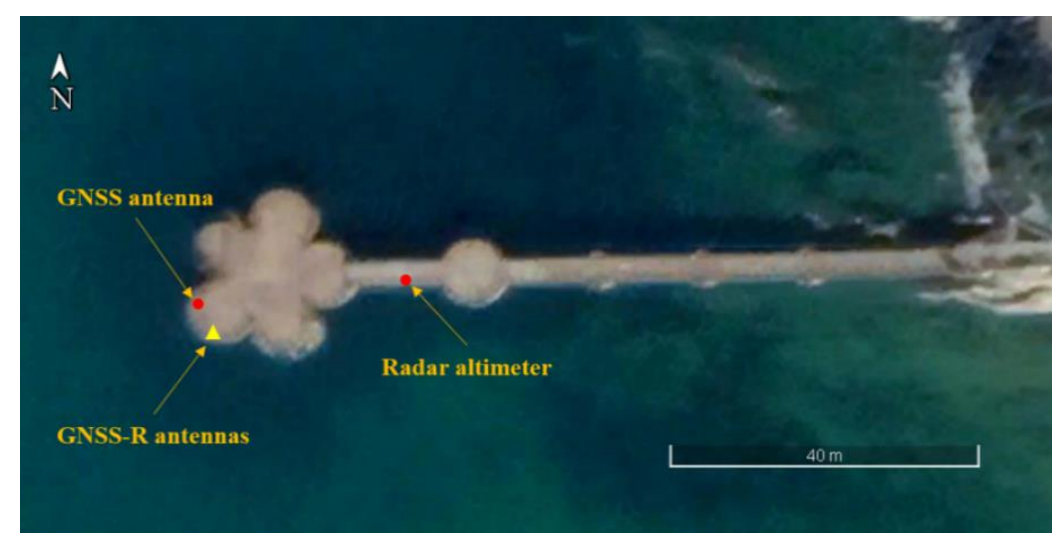

Figure 4. The top view of the experiment.

The GNSS-R antennas are mounted on a platform facing south to receive the QZSS GEO signals reflected off the sea surface as shown in Figure 5a. Both the dual-polarized GNSS-R antennas are identical. The operation band of the antenna is from 1.16 to $1.62 \mathrm{GHz}$. The antenna gain is $5 \mathrm{dBi}$ and the noise figure of the antennae is less than $1.5 \mathrm{~dB}$. The beam angle is $\pm 60^{\circ}$. The down-looking antenna is $30^{\circ}$ below the horizontal plane to receive the reflected signal, and the up-looking antenna is positioned symmetrically above the down-looking antenna to receive the direct signals. A recorder is connected to the antennas through RF lines and it digitizes the signals. Further, 2-bit real sampling is adopted. The IF data with the intermedia frequency of $15.58 \mathrm{MHz}$ are sampled at a rate of $62 \mathrm{MHz}$ and collected on a laptop computer. A GNSS receiver and a $26 \mathrm{GHz}$ radar altimeter are placed near the reflector, providing the in-situ SSH during the experiment, as shown in Figures $5 b$ and $6 c$. The height differences between the various equipment are measured accurately by a Total Station. A software-defined receiver (SDR) is developed to process 
the IF data. During the experiment, the sea surface is calm and the scatter is coherent. The code delay and phase delay measurements from the QZSS GEO satellite signals are both extracted.

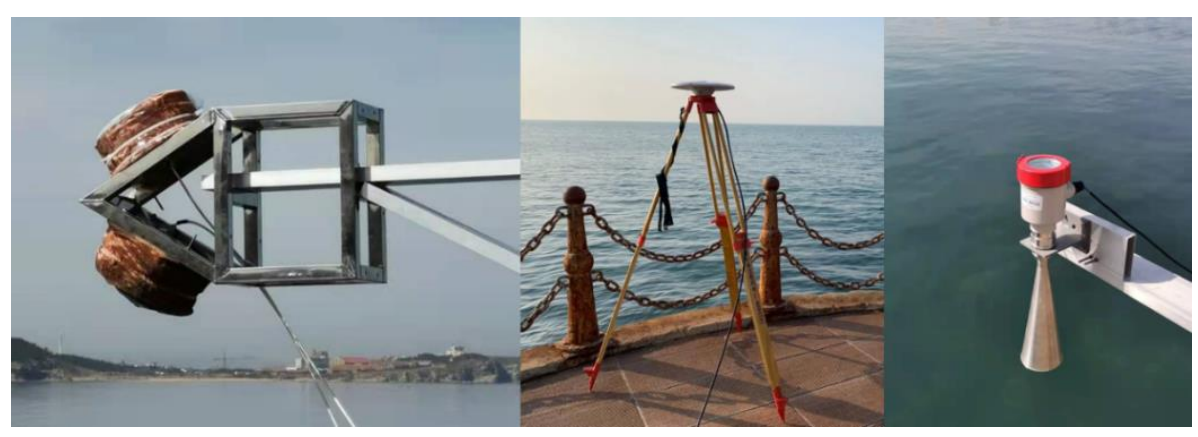

(a)

(b)

(c)

Figure 5. The instruments in the experiment. (a) the dual antennas; (b) the choke ring antenna for positioning; (c) the radar altimeter.

\subsection{Crosstalk Effect Simulation}

Based on (1) and (9), a simulation is carried out to analyze the impact of the crosstalk on the code delay measurements in the coastal condition. In order to simplify the simulation, L5 signal from QZSS GEO satellite is adopted to ensure high-precision code delay measurements, a long-term observation, and stable signal intensity. In addition, a better altimetry performance of L5 signal is expected because of the wide bandwidth and high transmission power, which is helpful to analyze the crosstalk. The code and phase path delay model is calculated using the in-situ SSH, the precise ephemeris from the IGS, and the precise position of the reflector.

The local waveform with a 2-chip-wide is generated by the correlation of the local L5 code. The local waveform is then lagged with the value of the code delay model to generate the waveform linked to $A_{r}^{2}$. The power waveforms associated with $A_{c}^{2}$ and $2 \cdot A_{c} \cdot A_{r} \cos (\delta \phi)$ are also generated using the same method. To determine the influence of the various parts on the code delay observations, three groups are settled to simulate the influence of crosstalk on the code delay measurements. Simulation I: $A_{r}^{2}+A_{c}^{2}$; simulation II): $A_{r}^{2}+2 \cdot A_{c} \cdot A_{r} \cos (\delta \phi)$; Simulation III: $2: A_{r}^{2}+A_{c}^{2}+2 \cdot A_{c} \cdot A_{r} \cos (\delta \phi)$.

In the simulation, the power ration $\frac{\mathrm{P}_{\mathrm{c}}}{P_{R}}$ determines the amount of the crosstalk signal entering the direct antenna, where $P_{c}$ is the power of the crosstalk signal and $P_{R}$ is the power of the reflected signal.

When the power ratio is $14 \%$, a constant bias between the simulation and delay model in simulation I is found and shown in Figure $6 a, b$ shows the results of simulation II. There is no obvious bias compared with simulation I, but a strong interference of a high-frequency term occurs. The high-frequency term worsens the code delay observation accuracy even without the bias. In the simulation, the scatter is coherent and the high-frequency part is related to the phase delay between the direct and reflected signals. When the scatter is non-coherent, a random high-frequency is observed and it reduces the precision of the measurements. The results of simulation III in Figure $6 \mathrm{c}$ demonstrate the real impact of the crosstalk on the code delay measurements in (9). With the parts related to $A_{m}^{2}$ and $2 \cdot A_{m} \cdot A_{r} \cos (\delta \phi)$, a bias and a high-frequency term appear simultaneously. Moreover, the precision of coastal GNSS-R altimetry using L5 signals is greatly reduced. Another power ratio is calculated as shown in Figure $6 \mathrm{~d}$, which indicates that the bias is proportional to the power ratio. The crosstalk can be reduced by the subtraction of the bias from the code delay measurements. 


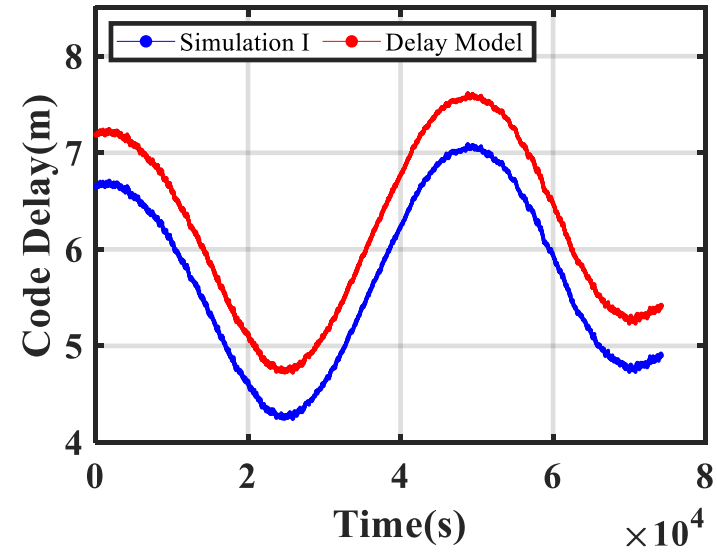

(a)

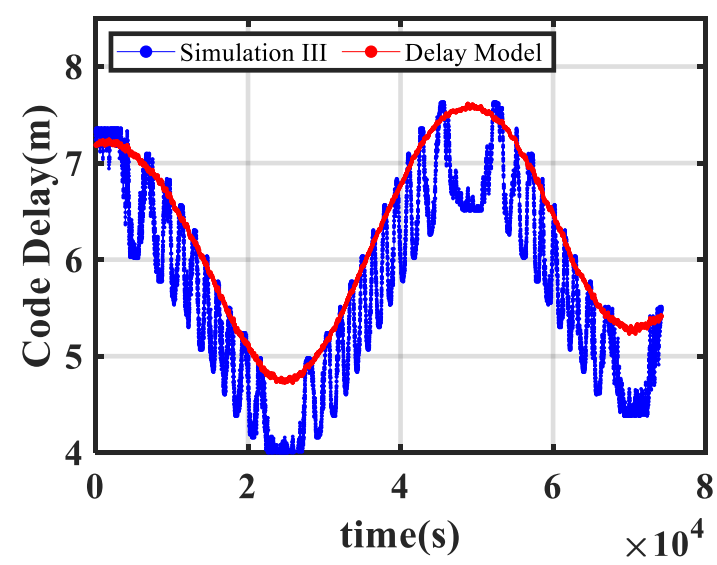

(c)

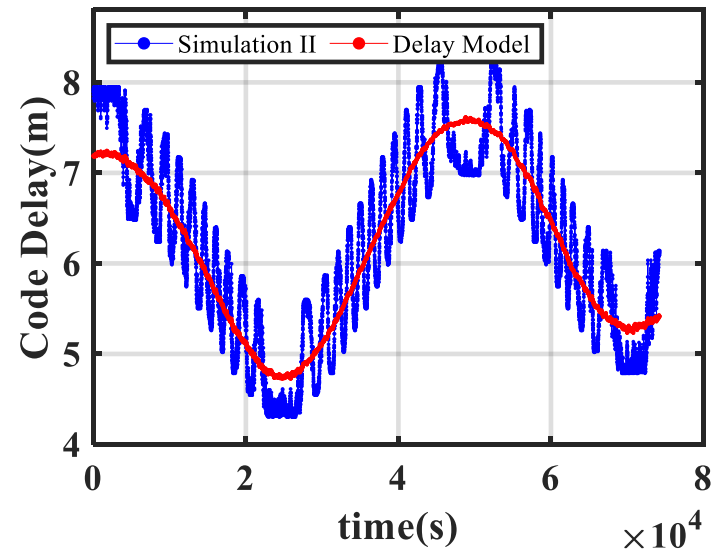

(b)

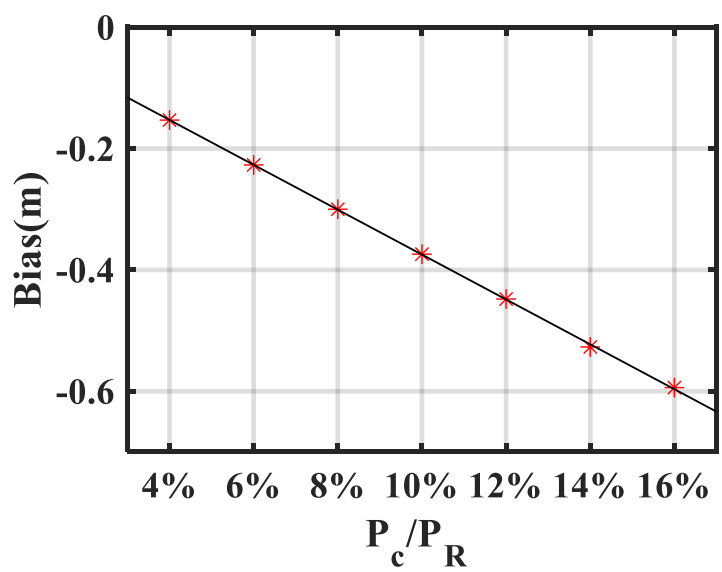

(d)

Figure 6. The simulation of the crosstalk impact on the code delay measurements: (a) The simulation I: $A_{r}^{2}+A_{c}^{2}$; (b) The simulation II: $A_{r}^{2}+2 \cdot A_{c} \cdot A_{r} \cos (\delta \phi)$; (c) The simulation III: $A_{r}^{2}+A_{c}^{2}+2 \cdot A_{c} \cdot A_{r} \cos (\delta \phi)$; (d) The relation between the power ratio of the crosstalk signal and the reflected signal with the measurements bias.

From the simulation, the impact of the crosstalk on the code delay measurements is depicted. Specifically, there is a bias related with the crosstalk signal amplitude and high-frequency term related with the interferometric phase between the direct and reflected signals. The coastal altimetry accuracy based on the code delay measurements would be decreased with the two factors.

\subsection{Crosstalk Effect in the Experiment}

The code delay measurements are extracted based on (12) and (13) using the SDR. Figure 7a shows the raw code delay measurements, the filtered code delay measurements, and the code delay model calculated from the in-situ SSH over approximately $23 \mathrm{~h}$ during the experiment. The code delay measurements output at $20 \mathrm{~Hz}$ sampling rate after Fourier interpolation in the SDR. Large errors caused by sea state, instrument, and other reasons are observed, and the precision of code delay measurements is significantly reduced. To filter errors, a moving mean filter of $60 \mathrm{~s}$ is used to obtain better measurements. The length of the filter window is an empirical value. Although a long time of the filter window may improve the accuracy, some information may be lost and it may affect further analysis. By comparing the filtered code delay measurements and the code delay model, a high-frequency term and a bias can be found in the experiment. 


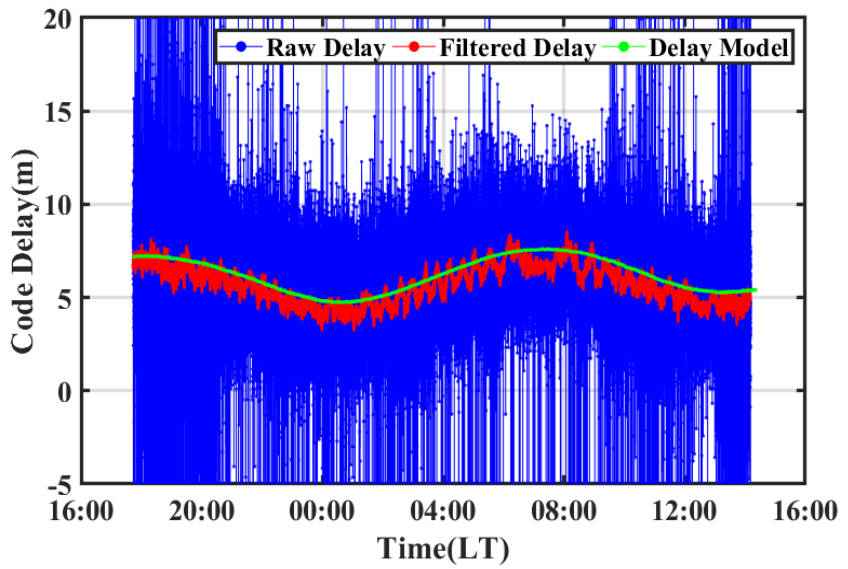

(a)

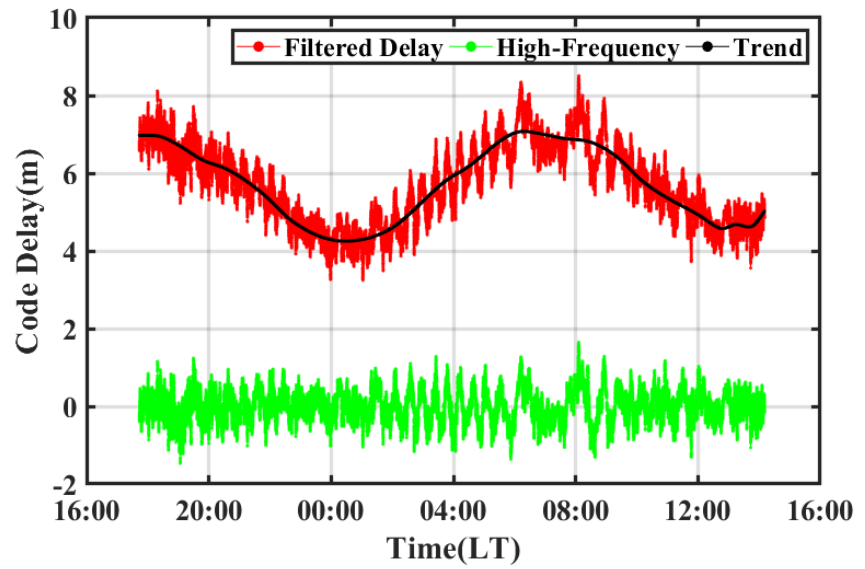

(b)

Figure 7. The code delay measurements during $23 \mathrm{~h}$ experiment: (a) The comparison between the raw code delay measurements (blue), the code delay measurements with 60-s moving mean filter (red), and the code delay model from the in-situ SSH (green); (b) The filtered code delay measurements with the EMD method: filtered measurements (red), the trend term (the black), the high-frequency term (green).

In order to analyze the high-frequency term and the bias, the empirical mode decomposition (EMD) method is used to decompose the filtered measurement sequences. The EMD method can be applied to the decomposition of nonstationary and non-linear data [37]. The GNSS-R water level monitoring accuracy can be effectively improved with the EMD method $[38,39]$. The filtered measurements are then decomposed and separated into 10 intrinsic mode function (IMF) components. The residual sequences are obtained by subtracting the sum of the IMFs from the filtered measurements. The residual sequences and the sum of the IMFs are the trend term and high-frequency term of the filtered measurements, respectively. Figure $7 \mathrm{~b}$ shows the trend term, the high-frequency term, and the filtered measurements. The sum of the IMFs has a mean value of approximately 0 , which means that the high-frequency term is extracted perfectly without bias. Compared with simulation II, a similarly strong interference of a high-frequency term is also found in the filtered measurements in the experiment. Moreover, this is an important reason for the decline in the precision of the coastal code altimetry when the random errors are filtered. Since the reason for the high-frequency term in simulation II is the phase difference between the direct and reflected signals, the same reason may also lead to the high-frequency term in the experiment.

The comparison of the high-frequency term and the phase delay in the experiment is shown in Figure 8a. In the coastal altimetry experiment, the scatter is coherent, as shown by the continuous phase delay measurements. The variation of the phase delay is completely compatible with the high-frequency term. The phenomenon can demonstrate that the phase delay between the direct and the reflected signals is the reason for the occurrence of the high-frequency term in the code delay measurements. According to simulation II, the part containing the phase information becomes important owning to the large power of the reflected signals even with the small power of the direct signal leaking into the reflected antennas, known as the crosstalk signal. Thus, the code altimetry performance is weakened as the induced high-frequency term. Further, a comparison of the trend term and the model is provided in Figure $8 \mathrm{~b}$. To contrast with simulation I, the obvious bias between the trend term and the in-situ measurements is observed despite some errors due to inappropriate decomposition of the EMD method, and the mean value of the bias is approximately $0.52 \mathrm{~m}$. As can be seen in Figure 6a, the power ratio between the crosstalk signal and the reflected signal is around $14 \%$, which demonstrates that the bias is due to the crosstalk signals. 


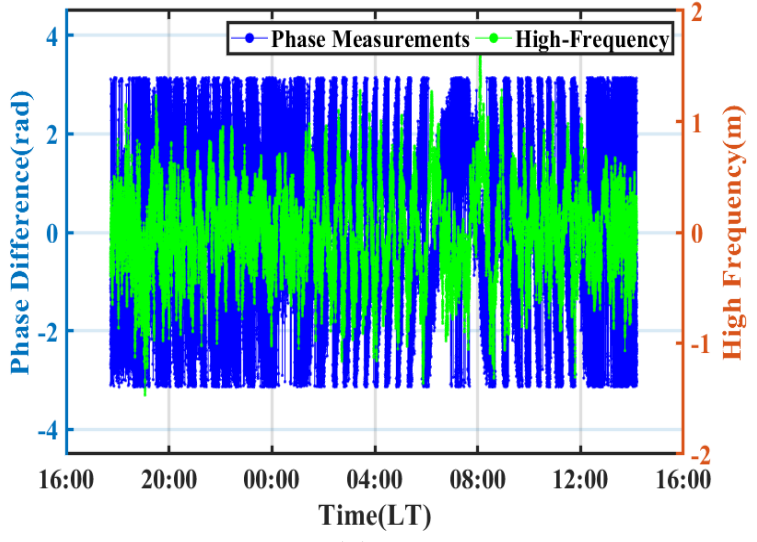

(a)

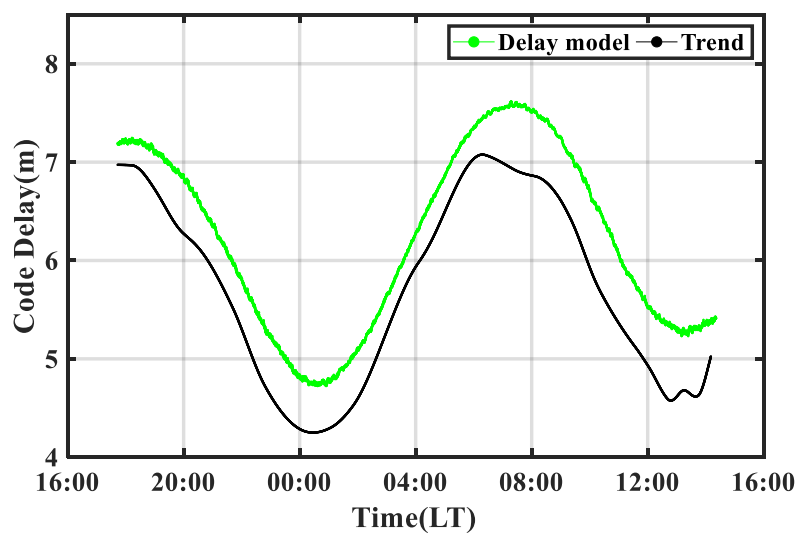

(b)

Figure 8. The results after the EMD method: (a) The comparison between the phase delay measurements (blue) and the high-frequency term (green); (b) The comparison between the trend (black) and the code delay model (green).

Figure 9 shows the comparison between simulation III and the code delay measurements in the experiment. In most of the periods, the variation of the measurements is consistent with the simulated measurements. In some periods, there are some differences in the high-frequency term becasue the phase delay model in the simulation is calculated from the in-situ SSH, which is different from the actual phase delay variation. The results can prove the existence of a crosstalk signal in the down-looking antenna.

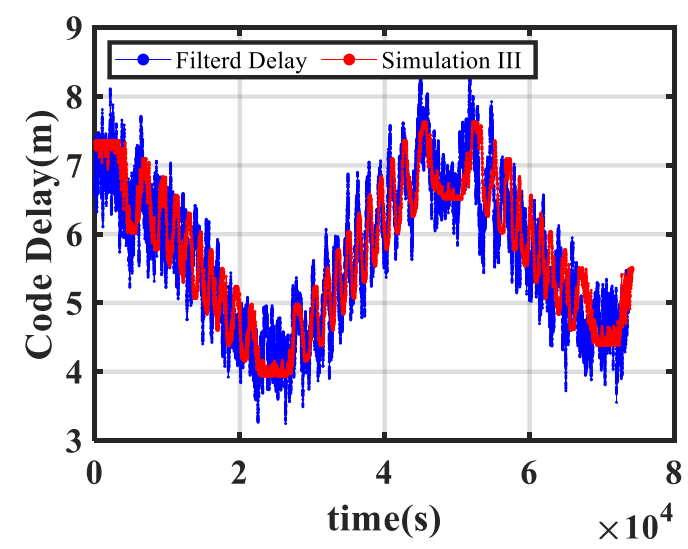

Figure 9. The comparison between the filtered delay measurements (blue) and simulation III (red).

\subsection{Crosstalk Effect Mitigation}

The high-frequency term induced by the phase delay and the trend term induced by the waveform distortion of the crosstalk represent the two reasons for the poor precision of the coastal code altimetry in GNSS-R. Thus, two different strategies are adopted. The EMD method is used to filter the high-frequency term and the power waveform is corrected to weaken the constant bias for the GEO satellite. Firstly, the standard power waveform is generated by using the local L5 code, and the power waveform of the crosstalk signal is built by adjusting the local power waveform value. Then, the power waveform of the crosstalk is subtracted from the waveform generated by the synthetic signals. Consequently, the two errors of code delay measurements can be corrected.

Figure 10 shows the correlation power of the direct and reflected signals during the experiment. As expected, there is no dramatic power average variation of the direct signal and reflected signal due to the stable geometry of coastal GNSS-R altimetry using GEO satellites. However, the small variation of the power indicates that the direct signals suffer heavily from the multipath effect, which has not been taken into account in the simulation for simplicity. On the other hand, the antennas cannot well suppress the interference in 
the experiment. Axelrad et al. (2005) proved that the code multipath error is proportional to the additional path length when the delay path length was shorter than half of the early/late discriminator spacing [40]. In our experiment, the maximum delay path length is about $14 \mathrm{~m}$, which is shorter than the discriminator spacing of $19.34 \mathrm{~m}$. As a result, the code phase of the direct signals is extended due to the path delay, and the path delay varies with the sea surface. When the reference signals are constructed, a 2-chip wide window is set with the direct signal code phase as the center, which causes the delay of the direct waveform. Therefore, the bias of the code delay measurements becomes larger. In order to realize the effective correction of the code delay observations, the power of the multipath signal is taken into account.

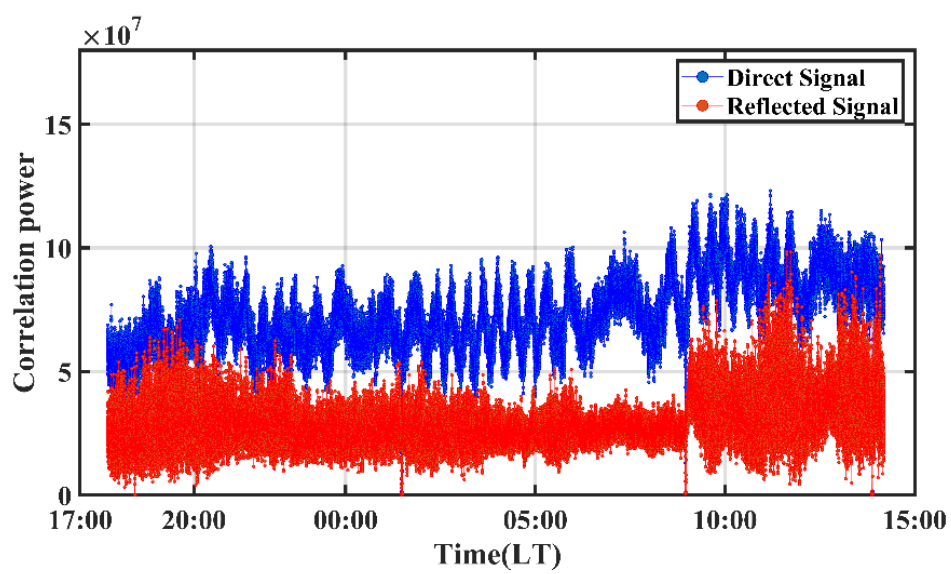

Figure 10. The power of the direct signals (blue) and the reflected signals (red).

As shown in the antenna patterns in Figure 11, the direct signal and the reflected signal will leak into antennas dedicated to the other signals. Furthermore, in the experiment, the antenna is symmetrically settled relative to the horizontal plane. The direct and the reflected signals will enter the antennas with the same antenna gain since the symmetric arrangement. Thus, the power of the crosstalk signal can be obtained by the direct signal power, and the power of the multipath signal can be obtained by the reflected signal power. The crosstalk power waveform is generated by using the sum of the crosstalk signals power and multipath signals power, which is then subtracted from the power waveform of the synthetic signals. As in the analysis, the bias can be weakened.

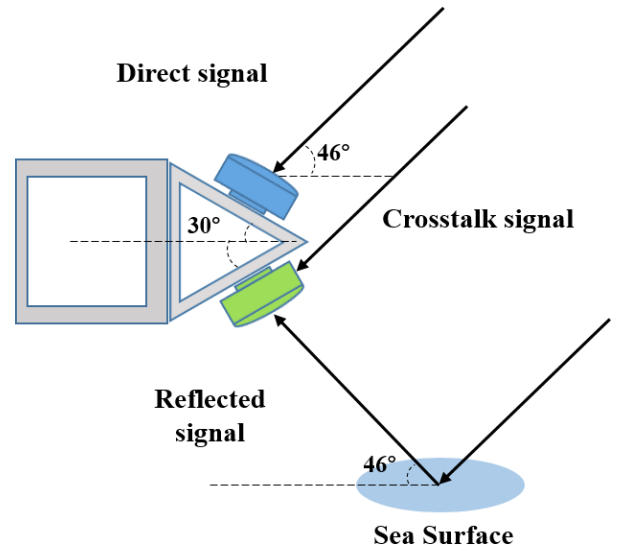

(a)

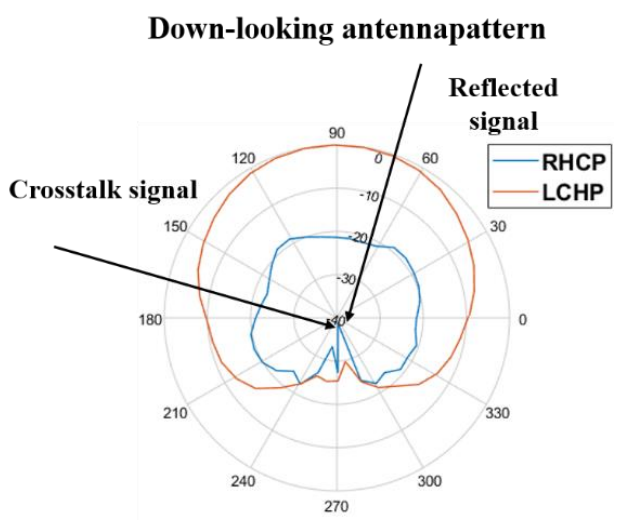

(b)

Figure 11. The sketch of the received signals: (a) the incident angle of the direct, reflected, and crosstalk signals, (b) the antenna pattern dedicated to the reflected signals. 
Table 1 shows the SSH precision calculated in the paper. According to the statistical results, coastal GNSS-R altimetry is greatly affected by the crosstalk signals, the large standard deviation (Std) is caused by the high-frequency term, and the large mean value (Mean) of the residual is caused by the bias term. Therefore, we reduce the high-frequency terms with different filtering methods. The bias is weakened by the waveform correction.

Table 1. The Std, Mean, and RMSE of the residuals between different methods and the in-situ SSH.

\begin{tabular}{cccc}
\hline Methods & Std (cm) & Mean (cm) & RMSE (cm) \\
\hline Code delay & 435.0 & -36.2 & 436 \\
Mean filter (1 min) & 30.1 & -35.2 & 46.3 \\
Mean filter(5 min) & 24.6 & -35.5 & 43.2 \\
EMD & 9.8 & -36.0 & 37.2 \\
Mean filter (1 min) (Bias removed) & 30.2 & -1.4 & 30.1 \\
Mean filter (5 min) (Bias removed) & 24.5 & -1.6 & 24.5 \\
EMD(Bias removed) & 9.3 & -1.8 & 9.5 \\
\hline
\end{tabular}

Before the bias is removed, the root mean squares error (RMSE) between the raw code delay measurements and the in-situ SSH is $436.0 \mathrm{~cm}$, which is useless in practice. With a moving mean filter of 1-min, most of the random errors can be filtered and the RMSE is $46.3 \mathrm{~cm}$. When the length of the filter window is enlarged to 5-min, the result is not obviously improved. When using a more appropriate filter, the code altimetry accuracy can be improved further. The SSH using the code delay measurements trend extracted by the EMD method shows that the Std is $9.8 \mathrm{~cm}$ and the RMSE is $37.3 \mathrm{~cm}$. The EMD method weakens the errors caused by the high-frequency term in the code delay measurements better than the mean filters. However, with a large mean value of approximately $35.0 \mathrm{~cm}$, the crosstalk is a critical problem in coastal code altimetry. After the correction of the power waveform, the bias is removed. The mean value of the residuals of $-1.4 \mathrm{~cm}$ and the RMSE of $30.1 \mathrm{~cm}$ is achieved using the 1-min filtered measurements. The 5-min mean filter could better filter the high-frequency term. With the EMD method, the trend term has an Std of $9.3 \mathrm{~cm}$. The RMSE between the trend of the measurements and the in-situ SSH is $9.5 \mathrm{~cm}$. The results show that the crosstalk is greatly mitigated by our method.

\section{Discussion}

Signal crosstalk would lead to a significant decline in the precision of code delay measurements. The crosstalk impact on the coastal GNSS-R code altimetry is analyzed in this paper. The L5 signal from QZSS GEO satellites is adopted in the experiment. With the mitigation of the crosstalk, the precision of SSH measurements can be improved under coastal conditions for a long time.

However, the multipath is not fully considered in the analysis because the multipath effect can be greatly reduced when the choke ring antenna is adopted. Moreover, if the antennas are far away from a reflective surface, the multipath effect on code delay measurements is more complicated. The mean filter is more suitable for continuously updated observations but poor to process the high-frequency term. Better filters still need to be studied. The bias can greatly weaken the coastal altimetry performance. For the GEO satellite, the bias is approximately a constant value and can be processed by the proposed method. The crosstalk impact on other types of GNSS satellites is more difficult than that of GEO. For example, the biases are different for each of the satellites and the high-frequency term will interfere with sea state and the other factors.

\section{Conclusions}

In this paper, L5 signals from the QZSS GEO satellite are used to study the impact of crosstalk signals on coastal GNSS-R code altimetry. Because the crosstalk signal leaks into the down-looking antenna, the power waveform is greatly changed according to the code altimetry principle. Through reasonable simplification, we simulate the impact of crosstalk 
on code delay observation. A constant bias and a high-frequency term are found. The bias is related to the amplitude of the crosstalk and the high-frequency term is related to the carrier phase delay between direct and reflected signals.

In order to verify our simulation, a coastal-based altimetry experiment is conducted. By comparing the simulation results with the measurements, the bias and the highfrequency term are also verified in the experiment. The high-frequency term contains the information of carrier phase delay and can be filtered out well by using the EMD method. The RMSE between the measurements and the in-situ SSH before removing the bias is $37.3 \mathrm{~cm}$. According to the analysis, we use the power of the direct and reflected signals to correct the power waveform of the synthetic signals. In addition, the mean value of the residual between the measurements and the in-situ SSH is $-1.8 \mathrm{~cm}$ after the crosstalk mitigation. At last, a 23-h-long coastal experiment demonstrates that the RMSE is about $9.5 \mathrm{~cm}$ when the bias is removed.

Author Contributions: Conceptualization, Y.H., T.X. and F.G.; methodology, Y.H.; software, Y.H., X.M. and F.G.; validation, N.W., X.M., Y.H. and B.N.; formal analysis, F.G.; investigation, F.G., X.M., Y.H. and B.N.; resources, F.G., X.M., Y.H. and B.N.; data curation, X.M., Y.H. and B.N.; writingoriginal draft preparation, Y.H.; writing—review and editing, Y.H., F.G., T.X. and N.W. All authors have read and agreed to the published version of the manuscript.

Funding: This research was jointly funded by the National Key Research and Development Program of China (2020YFB0505800 and 2020YFB0505804) and the Program of the National Natural Science Foundation of China (41604003, 41704017, 41704018).

Institutional Review Board Statement: Not applicable.

Informed Consent Statement: Not applicable.

Data Availability Statement: The datasets analyzed in this study are managed by Institute of Space Science, Shandong University and can be made available by the corresponding author on request.

Acknowledgments: The authors thank the staff of Weihai Golden Bay Hotel who kindly provided help during the experiment.

Conflicts of Interest: The authors declare no conflict of interest.

\section{References}

1. Melet, A.; Meyssignac, B.; Almar, R.; Le Cozannet, G. Under-estimated wave contribution to coastal sea-level rise. Nat. Clim. Chang. 2018, 8, 234-239. [CrossRef]

2. Liu, Y.; Kerkering, H.; Weisberg, R.H. Introduction to Coastal Ocean Observing Systems. In Coastal Ocean Observing Systems; Elsevier Inc.: Amsterdam, The Netherlands, 2015; pp. 1-10. ISBN 9780128020616.

3. Cazenave, A.; Cozannet, G. Le Sea level rise and its coastal impacts. Earth's Future 2014, 2, 15-34. [CrossRef]

4. Martin-Neira, M. A passive reflectometry and interferometry system (PARIS): Application to ocean altimetry. ESA J. 1993, 17, 331-355.

5. Löfgren, J.S.; Haas, R.; Scherneck, H.G. Sea level time series and ocean tide analysis from multipath signals at five GPS sites in different parts of the world. J. Geodyn. 2014, 80, 66-80. [CrossRef]

6. Wang, N.; Xu, T.; Gao, F.; Xu, G. Sea level estimation based on GNSS dual-frequency carrier phase linear combinations and SNR. Remote Sens. 2018, 10, 470. [CrossRef]

7. Wang, X.; He, X.; Zhang, Q. Evaluation and combination of quad-constellation multi-GNSS multipath reflectometry applied to sea level retrieval. Remote Sens. Environ. 2019, 231, 111229. [CrossRef]

8. Santamaría-Gómez, A.; Watson, C.; Gravelle, M.; King, M.; Wöppelmann, G. Levelling co-located GNSS and tide gauge stations using GNSS reflectometry. J. Geod. 2015, 89, 241-258. [CrossRef]

9. Larson, K.M.; Ray, R.D.; Williams, S.D.P. A 10-year comparison of water levels measured with a geodetic GPS receiver versus a conventional tide gauge. J. Atmos. Ocean. Technol. 2017, 34, 295-307. [CrossRef]

10. Larson, K.M.; Löfgren, J.S.; Haas, R. Coastal sea level measurements using a single geodetic GPS receiver. Adv. Space Res. 2013, 51, 1301-1310. [CrossRef]

11. Wu, J.; Chen, Y.; Gao, F.; Guo, P.; Wang, X.; Niu, X.; Wu, M.; Fu, N. Sea Surface Height Estimation by Ground-Based BDS GEO Satellite Reflectometry. IEEE J. Sel. Top. Appl. Earth Obs. Remote Sens. 2020, 13, 5550-5559. [CrossRef]

12. Martín-Neira, M.; Caparrini, M.; Font-Rossello, J.; Lannelongue, S.; Serra Vallmitjana, C. The PARIS Concept: An Experimental Demonstration of Sea Surface Altimetry Using GPS Reflected Signals. IEEE Trans. Geosci. Remote Sens. 2001, 39, 142-150. [CrossRef] 
13. Lowe, S.T.; Zuffada, C.; Chao, Y.; Kroger, P.; Young, L.E.; Labrecque, J.L. 5-cm-precision aircraft ocean altimetry using GPS reflections. Geophys. Res. Lett. 2002, 29, 13-1-13-4. [CrossRef]

14. Li, W.; Cardellach, E.; Fabra, F.; Ribo, S.; Rius, A. Assessment of Spaceborne GNSS-R Ocean Altimetry Performance Using CYGNSS Mission Raw Data. IEEE Trans. Geosci. Remote Sens. 2020, 58, 238-250. [CrossRef]

15. Gao, F.; Xu, T.; Wang, N.; He, Y.; Luo, X. A shipborne experiment using a dual-antenna reflectometry system for GPS/BDS code delay measurements. J. Geod. 2020, 94, 88. [CrossRef]

16. Rius, A.; Fabra, F.; Ribo, S.; Arco, J.C.; Oliveras, S.; Cardellach, E.; Camps, A.; Nogues-Correig, O.; Kainulainen, J.; Rohue, E.; et al. PARIS Interferometric Technique proof of concept: Sea surface altimetry measurements. In Proceedings of the 2012 IEEE International Geoscience and Remote Sensing Symposium, Munich, Germany, 22-27 July 2012; pp. 7067-7070. [CrossRef]

17. Cardellach, E.; Li, W.; Rius, A.; Semmling, M.; Wickert, J.; Zus, F.; Ruf, C.S.; Buontempo, C. First Precise Spaceborne Sea Surface Altimetry with GNSS Reflected Signals. IEEE J. Sel. Top. Appl. Earth Obs. Remote Sens. 2020, 13, 102-112. [CrossRef]

18. Pascual, D.; Park, H.; Onrubia, R.; Arroyo, A.A.; Querol, J.; Camps, A. Crosstalk Statistics and Impact in Interferometric GNSS-R. IEEE J. Sel. Top. Appl. Earth Obs. Remote Sens. 2016, 9, 4621-4630. [CrossRef]

19. Alonso-Arroyo, A.; Querol, J.; Lopez-Martinez, C.; Zavorotny, V.U.; Park, H.; Pascual, D.; Onrubia, R.; Camps, A. SNR and standard deviation of cGNSS-R and iGNSS-R scatterometric measurements. Sensors 2017, 17, 183. [CrossRef] [PubMed]

20. Lubeigt, C.; Ortega, L.; Vilà-Valls, J.; Lestarquit, L.; Chaumette, E. On the impact and mitigation of signal crosstalk in ground-based and low altitude airborne GNSS-R. Remote Sens. 2021, 13, 1085. [CrossRef]

21. Onrubia, R.; Pascual, D.; Park, H.; Camps, A.; Rüdiger, C.; Walker, J.P.; Monerris, A. Satellite cross-talk impact analysis in airborne interferometric global navigation satellite system-reflectometry with the microwave interferometric reflectometer. Remote Sens. 2019, 11, 1120. [CrossRef]

22. Gao, F.; Xu, T.; Meng, X.; Wang, N.; He, Y.; Ning, B. A Coastal Experiment for GNSS-R Code-Level Altimetry Using BDS-3 New Civil Signals. Remote Sens. 2021, 13, 1378. [CrossRef]

23. Kucwaj, J.C.; Stienne, G.; Reboul, S.; Choquel, J.B.; Benjelloun, M. Accurate Pseudorange Estimation by Means of Code and Phase Delay Integration: Application to GNSS-R Altimetry. IEEE J. Sel. Top. Appl. Earth Obs. Remote Sens. 2016, 9, 4854-4864. [CrossRef]

24. He, Y.; Gao, F.; Xu, T.; Meng, X.; Wang, N. Coastal Altimetry Using Interferometric Phase from GEO Satellite in Quasi-Zenith Satellite System. IEEE Geosci. Remote Sens. Lett. 2021, 1-5. [CrossRef]

25. Yang, Y.; Mao, Y.; Sun, B. Basic performance and future developments of BeiDou global navigation satellite system. Satell. Navig. 2020, 1, 1. [CrossRef]

26. Gao, F.; Xu, T.; Wang, N.; Jiang, C.; Du, Y.; Nie, W.; Xu, G. Spatiotemporal evaluation of GNSS-R based on future fully operational global multi-GNSS and eight-LEO constellations. Remote Sens. 2018, 10, 67. [CrossRef]

27. Wang, K.; Chen, P.; Zaminpardaz, S.; Teunissen, P.J.G. Precise regional L5 positioning with IRNSS and QZSS: Stand-alone and combined. GPS Solut. 2019, 23, 10. [CrossRef]

28. Nadarajah, N.; Khodabandeh, A.; Teunissen, P.J.G. Assessing the IRNSS L5-signal in combination with GPS, Galileo, and QZSS L5/E5a-signals for positioning and navigation. GPS Solut. 2016, 20, 289-297. [CrossRef]

29. Cabinet Office. Quasi-Zenith Satellite System Performance Standard (PS-QZSS-001); Cabinet Office: Tokyo, Japan, 2018 ; Volume 33.

30. Suzuki, T.; Kubo, N. GNSS-SDRLIB: An open-source and real-time GNSS software defined radio library. In Proceedings of the 27th International Technical Meeting of the Satellite Division of the Institute of Navigation (ION GNSS 2014), Tampa, FL, USA, 8-12 September 2014; Volume 2, pp. 1364-1375.

31. Zavorotny, V.U.; Voronovich, A.G. Scattering of GPS signals from the ocean with wind remote sensing application. IEEE Trans. Geosci. Remote Sens. 2000, 38, 951-964. [CrossRef]

32. Zavorotny, V.U.; Gleason, S.; Cardellach, E.; Camps, A. Tutorial on remote sensing using GNSS bistatic radar of opportunity. IEEE Geosci. Remote Sens. Mag. 2014, 2, 8-45. [CrossRef]

33. Liu, W.; Beckheinrich, J.; Semmling, M.; Ramatschi, M.; Vey, S.; Wickert, J.; Hobiger, T.; Haas, R. Coastal Sea-Level Measurements Based on GNSS-R Phase Altimetry: A Case Study at the Onsala Space Observatory, Sweden. IEEE Trans. Geosci. Remote Sens. 2017, 55, 5625-5636. [CrossRef]

34. Yu, K.; Li, Y.; Chang, X. Snow Depth Estimation Based on Combination of Pseudorange and Carrier Phase of GNSS DualFrequency Signals. IEEE Trans. Geosci. Remote Sens. 2019, 57, 1817-1828. [CrossRef]

35. Yu, K.; Ban, W.; Zhang, X.; Yu, X. Snow depth estimation based on multipath phase combination of GPS triple-frequency signals. IEEE Trans. Geosci. Remote Sens. 2015, 53, 5100-5109. [CrossRef]

36. Nievinski, F.G.; Larson, K.M. Forward modeling of GPS multipath for near-surface reflectometry and positioning applications. GPS Solut. 2014, 18, 309-322. [CrossRef]

37. Huang, N.E.; Shen, Z.; Long, S.R.; Wu, M.C.; Snin, H.H.; Zheng, Q.; Yen, N.C.; Tung, C.C.; Liu, H.H. The empirical mode decomposition and the Hubert spectrum for nonlinear and non-stationary time series analysis. Proc. R. Soc. A Math. Phys. Eng. Sci. 1998, 454, 903-995. [CrossRef]

38. Beckheinrich, J.; Hirrle, A.; Schön, S.; Beyerle, G.; Semmling, M.; Wickert, J. Water level monitoring of the Mekong Delta using GNSS reflectometry technique. In Proceedings of the 2014 IEEE Geoscience and Remote Sensing Symposium, Quebec City, QC, Canada, 13-18 July 2014; pp. 3798-3801. 
39. Zhang, S.; Liu, K.; Liu, Q.; Zhang, C.; Zhang, Q.; Nan, Y. Tide variation monitoring based improved GNSS-MR by empirical mode decomposition. Adv. Space Res. 2019, 63, 3333-3345. [CrossRef]

40. Axelrad, P.; Larson, K.; Jones, B. Use of the correct satellite repeat period to characterize and reduce site-specific multipath errors. In Proceedings of the 18th International Technical Meeting of the Satellite Division of The Institute of Navigation (ION GNSS 2005), Long Beach, CA, USA, 13-16 September 2005; pp. 2638-2648. 